\title{
STUDY ON THE EVOLUTION, RESULTS AND TRENDS OF APPLYING STOCHASTIC OPTIMIZATION
}

\author{
Alexandru HAMPU, Vasile CĂRUȚAȘU \\ "Nicolae Bălcescu” Land Forces Academy, Sibiu, Romania \\ alexhampu@yahoo.com, v.carutasu@yahoo.com
}

\begin{abstract}
This paper aims to analyze the stage reached in the development and application of stochastic optimization models, highlighting some of the most important moments and achievements in the field. The author tries to identify particular aspects that define the classes of stochastic optimization models, specifying the level reached in certain directions of research and implementation of these models in order to identify possible directions of development of these specific techniques.
\end{abstract}

\section{Keywords: Stochastic optimization}

\section{Evolution of stochastic optimization} Stochastic Linear Programming (Optimization) is a branch of mathematical programming that emerged from the need for decision-making optimization in processes or activities where some intervening factors are random.

Starting from the model of the deterministic mathematical programming, a multitude of stochastic models have been made which preserve their general structure but have acquired a particular character taking into account the characteristic conditions of each model. A general form of astochastic programming problem (SPP) is:

$\min (\max ) z(x, \omega)=c^{\mathrm{T}}(\omega) x(1)$

subject to: $\left\{\begin{array}{c}A(\omega) x=b(\omega) \\ x \geq 0,\end{array}\right.$

where $A(\omega), \quad b(\omega), \quad c(\omega)$ are random matrices of dimensions $A: m \times n, b: m \times 1, c$ : $n \times 1$ whose elements are random variables defined on the probability field $\{\Omega, K, \mathrm{P}\}$.

Noting $X(\omega)=\left\{x \in \mathbb{R}^{n} \mid A(\omega) x=b(\omega), x \geq\right.$ $0\}$, the problem (1.1)-(1.2) becomes:

$$
\max z(x, \omega)==c^{\mathrm{T}}(\omega) x(3) \text { subject }
$$

to: $x \in X(\omega)$

According to some authors a classification of stochastic models can be made from the two principles formulated in (SPP): "wait and see" respectively "here and now". For the first case where a (SPP) issolved as a deterministic one that has as a characteristic a probability distribution, it is called distribution problems. For the second case, we mention two of the main types of problems, namely Two-Stage Programming Problem and Programming under Probabilistic Constraint.

\subsection{Distribution Problems}

If $z(x, \omega)$ of relation (1) is a random variable, it is not possible to know the values of this function before determining the exact values of the coefficients, which is why we try to determine the distribution function and / or some characteristic values such as the mean or the dispersion. The first who formulated this problem was $G$. Tintnerin the work [20]. This type of 
problem is also known as "wait and see", named by A. Mandansky.

Let us consider the problem (SPP) (3) -(4); if $(A, b, c)$ has a known distribution it appears to be a deterministic problem. However, we can have situations of different types: it is possible that only the objective function is a random vector, only $b$ is random, $A$ is random or there are combinations of the three situations.

If only $c$ is random vector and the set of possible solutions $X=\left\{x \in \mathbb{R}^{n} \mid A x=b, x \geq 0\right\}$

has constant values, then one of the extreme points of the convex set $X$ will be the optimal solution.In such a situation the random vector $c$ can be written in the form $c^{\mathrm{T}}+t(\omega) \cdot d^{\mathrm{T}}$ the objective function being $z(x, \omega)=\left(c^{\mathrm{T}}+t(\omega) d^{\mathrm{T}}\right) x$ and the distribution function $F(z)$ of the random variable $\mathrm{z}$ is like:

$$
\begin{gathered}
\mathrm{F}(\mathrm{z})=\sum_{j=1}^{p} P\left\{\left[\omega \mid\left(c^{\mathrm{T}}+t(\omega) d^{\mathrm{T}}\right) x^{j}<\right.\right. \\
\left.<z \bigcap\left[\omega \mid \lambda_{j-1} \leq t(\omega)<\lambda_{j}\right]\right\}=\sum_{j=1}^{p} H_{j}(z)
\end{gathered}
$$

For this case of the vector $\mathrm{z}$ important results were obtained by B. Bereanu [2]; for the general case in which $z$ is random without the previous form J.B. Ewbank, B.L. Foote and H.J. Kumin[10] have determined the distribution function with a certain type of integrals.

If the vector $b$ is random the problem can be solved by using the dualproblem as in the random case of $c$. Important results were obtained by B. Bereanu [3] who formulated three theorems related to the continuity of the optimal values of the respective model. Another method that can be used in this case in which only $c$ is random is the simplex algorithm.

There are different methods for determining the distribution function; some are exact but very laborious methods, others are methods that approximate the distribution function.From the first category we can remember the complete description method and as approximate methods: the simulation method, the discretization method, the incomplete description method, the Cartesian integration method [3]. Some of these methods are described in [17].

If the vectors $c$ and $b$ are random, the simulation method can also be used. We note with $\mu(A, b, c)$ the optimum value of the problem, we assume there is a lot of possible solutions, $\mu$ is finite with probability 1 and its mean exists. Then we can generate values (samples) $\left(A_{i}, b_{i}, c_{i}\right)$, $i=1, \ldots, N$ independent which are nonrandom and approximate $E(\mu)$ in the form:

$$
\mu_{\mathrm{N}}=\frac{1}{N} \sum_{i=1}^{N} \mu\left(A_{i}, b_{i}, \mathrm{c}_{i}\right)
$$

and the simulation method is used to calculate $P(\mu \leq z)$.

The discretization method can be applied to a problem where $A, b, c$ are partially or entirely random and $H$ and $h$ are deterministic values with which deterministic restrictions $H x \leq h$ are formed other than random $A x \leq b$.

Assuming that $(A, b, c)$ has a finite number of possible values $\left(A^{(i)}, b^{(i)}, c^{(i)}\right), i==1,2, \ldots$, $s$ which occur with the probabilities $\mathrm{p}_{\mathrm{i}}$, the optimal value of the linear pro-gramming problemcan be calculated [13]:

$\max z=c^{(i) \mathrm{T}} x$

subject to: $H x \leq h(5)$

$$
\begin{aligned}
& A^{(i)} x \leq b^{(i)} \\
& x \geq 0
\end{aligned}
$$

for $i=1, \ldots, t$.If $x^{(i)}$ is an optimal solution of the problem (5), and considering the optimum of the objective function $\mu^{(i)}==c^{(i) \mathrm{T}} x^{(i)}$ then the mean of the function is:

$E(\mu)=\sum_{i=1}^{r} p_{i} \mu^{(i)}$

For a $c$ deterministic and if we have a small number of restrictions in the problem (5) we first solve the type $H x \leq h$ then the restrictions $A^{(i)} x \leq b^{(i)}$ for each $i, i=1,2, \ldots, s$.

The last method in the approximate category is the Cartesian integration methodintroduced by B. Bereanu [4]. Random matrices can be represented as linear functions, as follows:

$$
A=A^{(0)}+A^{(1)} \omega_{1}+\ldots+A^{(s)} \omega_{s}
$$




$$
\begin{aligned}
& b=b^{(0)}+b^{(1)} \omega_{1}+\ldots+b^{(s)} \omega_{s} \\
& c=c^{(0)}+c^{(1)} \omega_{1}+\ldots+c^{(s)} \omega_{s}
\end{aligned}
$$

If certain conditions are met by $\omega$ formulated in [4], the mean $E(\mu(\xi))$ can be calculated:

$E(\mu(\omega))=\int_{T} \mu(v) \varphi(v) d v(7)$

where it was considered $v=\left(v_{1}, v_{2}, \ldots, v_{s}\right)$ instead of $\omega$, and the notation $A(v), b(v)$, $c(v)$ expresses the dependence of vectors $A$, $b, c$ on $v$; by $\varphi(v)$ we have designated the probability density of $\omega, \mu(\omega)$ will be noted with $\mu(v)=\mu(A(v), b(v), c(v))$ being a continuous function in $T$.

Bereanu used a formula called the Cartesian multidimensional quadrature formula, i.e. an approximation sum for the integral (7). The form of this quadrature formula introduced in [19] is:

$$
L_{n}^{s}(f)=\sum_{i_{1}=1}^{n} \sum_{i_{s}=1}^{n} c_{1, i_{1}}^{(n)} \ldots c_{s, i_{l_{s}}}^{(n)} f\left(x_{1, i_{1}}^{(n)} \ldots x_{s, i_{l_{s}}}^{(n)}\right)(8)
$$

Using such a formula, the integrals of type (7) as well as other forms, the objective function can be calculated.

\subsection{Two-stage stochastic programing problems}

In such (SSP) decision will be taken in two stages; given the existence of some restrictions in which $b$ is random, in this case it will not be possible to determine $x$ so that the relation $A x=b$ will occur. There will be a difference between the left and right members which will be a random variable that will cause the penalty of the objective function, a penalty that must obviously be minimized. The general model introduced by Beale [1] and Dantzig [8] is:

subject to $: A x+B y=b$

$$
\min \left\{c^{\mathrm{T}} x+\mathrm{E}\left(\min _{y} q^{T} y\right)\right\}
$$

$$
x, y \geq 0
$$

the second stage (the recourse problem) being as follows:

$$
\min q^{\mathrm{T}} y
$$

subject to: $B y=b-A x$

$y \geq 0$.

Obtaining the minimum of the second stage $Q(x, b)$ its mean value is determined
$Q(x)=\mathrm{E} Q(x, b)$ the stochastic case becoming a deterministic one.

Some important results related to the twostage stochastic programming problems refer to the submodels derived from it both as a form of expression and as solving methods. G.B. Dantzig [8], E.M.L. Beale [1], and R. Wets [21] have studied this model, which they calledthe complete recourse, considering that the matrix can be partitioned into form $B=(I,-I)$, where $I$ is the unit matrix. If $B=I$ the model is called with a simplerecourse.

For the stochastic programming with recourse, different approaches have been used depending on the conditions set for the problem and for which there are different solving techniques:

- primal method proposed in 1983 by Wets [22]. The algorithm uses a base called a perfect basis or a workbasis, whose existence is demonstrated and which allows to determine the optimal solution.

- dual method proposed byPrékopa [14]in 1990 based on Lemke's simplex dual algorithm [12] in 1954.

- decomposition stochasticmethod if $\xi$ (the random vector of free terms) has a discrete distribution that was initiated by Strazicky [19]. The author uses a particular pattern structure that allows the dual problem writing so that the revised simplex algorithm can be used. The generalized case of the algorithm can be used if both $\xi$ and $B$ or $q$ are random with a finite number of realizations.

-L-shapedmethod introduced by Van Slyke and Wets [16] resolves stochastic programming problems with recourse and optimal control problems by means of an algorithm that generates linear restrictions for those restrictions that are violated by a decision vector that is supposed to be optimal. The form of an L-shaped program is the following:

$\min z=c^{1} x+c^{2} y(8)$

subject to: $A^{11} x \quad=b^{1}(9)$

$A^{21} x+A^{22} y=b^{2}(10)$

$x \geq 0, y \geq 0$ 
The use of the program is made taking into account some of the features that it needs to have, namely:

(i) the restrictions (10) are formulated in the sense that for those vectors $x$ for which $A^{11} x$ $=b^{1}, x \geq 0$, there exists $y \geq 0 A^{21} x+A^{22} y=b^{2}$ are satisfied.

(ii) the vector $y$ and $c^{2} y$ are relatively small factors in determining the optimum value

(iii) the restrictions (10) are large in number, possibly infinite.

The model is resolved by a three-step algorithm using the simplex algorithm. It is also presented the possibility of approach by decomposition method due to Dantzig and Wolfe [9].

Another form of two-stage stochastic programming model is the integer stochastic programming in two stages in which some or all of the variants that appear in the problem are integers and are supposed to be discrete. The model was introduced by R.M. Wollmer [23] and has at least two approaches to solving:

- the first one relieson approximate the optimal solution through various probabilistic methods approaching by L. Stougie [18].

- the second way of solving is based on the Benders decomposition through which the optimal determination is made.

\subsection{Programming under probabilistic constraint}

Programming under probabilistic constraint appeared due to some stochastic programming problems of certain restrictions which can not be known with certainty, in which case a threshold $\propto$ is established over which to obtain the probability of realization of the restrictions. optimum $z=c^{\mathrm{T}} x$

subject to: $\quad P(A x \leq b) \geq \propto$

$x \geq 0$,

where $\propto$ is a probability vector.

In such a model, some restrictions may be deterministic.

The model was introduced by Charnes, Cooper and Symonds [6] under the name of chance constrained programming and later developed by other authors.

One approach is to make the decision after the stochastic problem transforms into what is called its deterministic equivalent [6]. It is a method of transforming probability constraints into some deterministic ones, and it is particularly interesting where the deterministic equivalent leads us to convex programming that can be solved by specific methods. Programming under probabilistic constraint contains a relatively large number of models, of which we will list only a few:

-E- model:

$\max \mathrm{E}\left(c^{\mathrm{T}} x\right)$

subject to: $P(A x \leq b) \geq \propto$

$x=D b$

where $\mathrm{P}$ is a probability, $\mathrm{E}$ is the expected operator $A, b$ and $c$ may have some or all of the random components

- $V$ - model: $\min \mathrm{E}\left(c^{\mathrm{T}} x-c^{0 \mathrm{~T}} x^{0}\right)^{2}$

subject to: $P(A x \leq b) \geq \propto$

$x=D b$

where the objective function is to minimize the error caused by the deviation from a preferred value $z^{0}=c^{0 \mathrm{~T}} x^{0}$.

- $P$ - model:

$$
\max P\left(c^{\mathrm{T}} x \geq c^{0 \mathrm{~T}} x^{0}\right)^{2}
$$

subject to: $P(A x \leq b) \geq \propto$

$x=D b$.

These three models are representative for programming under probabilistic constraint, some particular cases existing besides them.

\section{Trends in the evolution of stochastic programming}

We will further present some trends in the (SPP) approach over the last few years and will most likely notice the same trend.

The main issues regarding the current trends in the stochastic programming approach are based on the following causes which obviously imply certain directions of evolution (SPP):

a) Incomplete deciphering of the internal structure of existing models, of some of their properties, which will further determine studies on the establishment of 
all the theoretical aspects of these models. There are still such situations where not all properties, valences, or usage limits of their models are known.

b) Lack of or insufficient development of models to optimize certain activities. Generally, optimization models and techniques for most of the stochastic processes have been developed, but there are situations where no such models have yet been made, the causes being diverse, most often related to the difficulty of approach. For those classes of activities or processes for which (SPP) models have not yet been developed, new research directions will obviously be opened to lead to the associated models.

c) The absence of software based on certain models already created, or the existence of barriers to their realization. This causes a trend that seems to be the most obvious because it takes into account the practical side of using stochastic programming as a working tool of the decision maker. An example of this is the computational aspect of stochastic programming, which often leads to the approximation of a large number of possible scenarios by its significant reduction, which is imposed by the limited capacities of ordinary computers. Methods to solve this problem are sought, for example the stochastic decompostation proposed in the [15] paper which for a class of problems (SPP) allows the decision to be called compromise, a new concept of decision. The method provides a solution similar to the classic method but with a much smaller amount of computing that can be done on common computers. In recent years, there has been a trend towards the development of models that can be run through software designed for office calculators available to decision-makers, with emphasis on ease of use by decision makers who are not very well-advised in the field. There are complex software such as XPRESS orAIMMS that include (SPP) and are used in major companies as well as in Universities to solve some of the most diverse practical applications or current decision making optimizations.

\section{Conclusions}

In this paper I have analyzed some of the main models (SPP) as well as some trends in the evolution of studies in this field.

It is worth pointing out that after 1950, when these techniques were used to optimize decision-making in various areas of human activity, the benefits of using such optimization methods were obvious.

In astochastic economic, industrial, military etc. activity involving relatively large financial, material or other expense, the optimal decision can only be obtained on the basis of decision-making techniques based on, among other things, models of stochastic programming. There are known situations where decisions have been made on the basis of stochastic modeling in large-scale investments, a well-known example of the regulation of Lake Balaton based on the models applied by Prékopa and Szántai, modeling which yielded excellent results proven practically in the 40 years of exploitation, this being an expression of the effectiveness of the decision in this economic activity involving major expenditure.

The conclusion is that, being a very useful tool for decision making in the case of random activities, stochastic programming will continue to evolve by identifying new directions and possibilities for application.

\section{References}

[1] Beale, E.M.L., On Minimizing a Convex Function Subject to Linear Inequalities, Journal of the Royal Statistical Society. Series B ,vol 17 (2), pp.173-184, 1955.

[2] Bereanu, B.,On Stochastic Linear Programming. Distributions Problems, Stochastic Technology Matrix, Z. Wahrscheinlichkeitstheorie Verw. Gebiete, 8, pp.148-152, 1967.

[3] Bereanu, B., The Continuity of the Optimumin Parametric Programming and Applications to Stochastic Programming, J. of Opt. Theory and its Applications 18, pp.319-333, 1976. 
[4] Bereanu, B.,The Distribution Problem in Stochastic Linear Programming. The Cartesian Integration Method, Reprint No. 7103, Center of Mathematical Statistics of the Academy of the Socialist Republic of Romania, Bucharest, pp.71-103, 1971.

[5] Bereanu, B.,Some Numerical Methods in Stochastic Linear Programming under Risk and Uncertainty, Stoch. Programming (M.A.H. Dempster, ed), Academic Press, London, pp.196-205, 1980.

[6] Charnes, A., Cooper, W.W. și Symonds, G.H. Cost Horizons and Certainty Equivalents: an Approach to Stochastic Programming of Heating Oil, Management Sci., 4, pp.235-263, 1958

[7] Charnes, A., Cooper, W.W., Deterministic Equivalents for Optimizating and Satisfying under Chance Constraint. , Operation Research 11(1), pp.18-39, 1963.

[8] Dantzig, G.B.,Linear Programming under Uncertainty, Management Sci., 1, pp.197206, 1955.

[9] Dantzig, G.B. and Wolfe,P., The DescompositionAlgorithm for Linear Programs, Econometrica, vol.29, pp.767-778, 1961.

[10] Ewbank, J.B., Foote, B.L., Kumin. H.J., A Method for the Solution of the Distribution Problem of Stochastic Linear Programming, SIAM J. Appl. Math., 26, pp.237-243, 1974.

[11] Lageweg, B.J., Lenstra, J.K.,Rinnoy Kan, A.H.G.R., Stougie, L., Stochastic Integer Programming by Dynamic Programming, StatisticaNeerlandica, 39 (2), 97-113, 1985.

[12] Lemke, C.E., The Dual Method for Solving the Linear Programming Problem, Naval Research Logistic Quarterly 1, pp.36-47, 1954.

[13] Prékopa, A., Stochastic Programming, AkadémiaKiadó, Budapest, 1995.

[14] Prékopa, A., Dual Method for a One-stage Stochastic Programming Problem with Random RHS Obeying a Discrete Probability Distribution, Zeitschrift fur Op.Research, 38, pp.441-461, 1990.

[15] Sen, S. and Liu, Y., Mitigating Uncertainty via Compromise Decision in Two-stage Stochastic Linear Programming,Operations Research 64 (6), 1422-1437, 2014.

[16] Slyke, R,. van, Wets, R.J.-B., L-shaped Linear Program with Applica.tion to Optimal Control and Stochastic Linear Programs, SIAM J. on Applied Math. 17, pp.638-663, 1969

[17] Stancu-Minasian, I.M.,Programareastochastică cu mail multefuncțiiobiectiv, Ed. AcademieiRSR, 1980.

[18] Stougie, L., Design and Analysis of Algorithms for Stochastic Integer Programming, CWI Tract 37, Centrum voorWiskundeen Informatica, Amsterdam, 1987.

[19] Strazicky, B., Computational Experience with Algorithm for Discrete Recourse Problems, Stochastic Programming (M.A.H. Dempster, ed.) Academis Press, London, pp.263-274, 1980.

[20] Tintner, G., Stochastic Linear Programming with Applications to Agricultural Economicsz, Proseedings of the Second Symposium in Linear Programming, H.A. Antotosiewicz (ed.) National Bureau Standard, Washington, pp. 197-228, 1955.

[21] Wets, R.J.-B., Programming under uncertainty: the complete problem, Z. WahrscheinlichkeitstheorieVerw, Gebiete, 4, pp.316-339, 1966.

[22] Wets, R.J.-B., Solving stochastic programs with simple recourse, Stochastics 10, pp.219-242, 1983.

[23] Wollmer, R.M., Two-stage linear programming under uncertainty with 0-1 first stage variables, Mathematical programming 19, pp.279-288, 1980.

www.aimms.com.

https://www.solver.com/xpress-solver-engine 